\title{
Identification of responders/non-responders to 5-fluorouracil based on individual $50 \%$ inhibitory area under the concentration curve of 5 -fluorouracil obtained with collagen gel droplet-embedded culture-drug sensitivity test in colorectal cancer
}

\author{
TAKUMI OCHIAI ${ }^{1}$, KAZUHIKO NISHIMURA ${ }^{1}$, TOMOO WATANABE ${ }^{1}$, MASAYUKI KITAJIMA ${ }^{1}$, \\ AKINORI NAKATANI ${ }^{1}$, TAKASHI MARUSASA ${ }^{1}$, TADASUKE HASHIGUCHI ${ }^{1}$, TAKAYUKI UCHIDA ${ }^{1}$, \\ NAOKI SAKUYAMA ${ }^{1}$, TSUYOSHI SATO ${ }^{1}$, KENNJI KISHINE ${ }^{1}$, SHUNJI FUTAGAWA ${ }^{1}$ and ISAO NAGAOKA ${ }^{2}$ \\ ${ }^{1}$ Department of Surgery, Tobu Chiiki Hospital, Tokyo Metropolitan Health and Medical Treatment Corporation; \\ ${ }^{2}$ Department of Host Defense and Biochemical Research, Juntendo University School of Medicine, Tokyo, Japan
}

Received October 5, 2010; Accepted December 22, 2010

DOI: $10.3892 / \mathrm{ol} .2011 .251$

\begin{abstract}
We previously reported the 5-fluorouracil (5-FU) sensitivity of cancer cells obtained from colorectal cancer (CRC) patients using the collagen gel droplet-embedded culturedrug sensitivity test (CD-DST). Multiple drug concentrations and contact durations, and the area under the concentration curve (AUC) and growth inhibition rate (IR) were combined, resulting in the AUC-IR curve, which was approximated to the logarithmic curve. Moreover, the individualized $\mathrm{AUC}_{\mathrm{IR} 50}$, the AUC value which gives 50\% growth inhibition, was calculated using the AUC-IR curve. This study aimed to identify responders/non-responders to 5-FU based on the individual $\mathrm{AUC}_{\text {IR50 }}$ obtained with CD-DST in order to establish individualized chemotherapy for CRC patients. The individual $\mathrm{AUC}_{\mathrm{IR} 50}$ was calculated from each AUC-inhibition rate regression curve in all patients using the CD-DST. The cumulative distribution of the individual $\mathrm{AUC}_{\mathrm{IR} 50}$ in CRC patients was evaluated. The cumulative distribution of the individual $\mathrm{AUC}_{\mathrm{IR} 50}$ was regressed over the sigmoid curve (logarithmic scale). The approximate expression was almost exactly $\mathrm{y}=\mathrm{ab}^{\wedge} \exp (-\mathrm{cx}) \quad(\mathrm{a}=0.9739$, $\mathrm{b}=1.7096 \mathrm{E}-21, \mathrm{c}=0.8990$, the sum of square residuals, 0.0279). In the 80 cases examined, no notable change was observed in
\end{abstract}

Correspondence to: Dr Takumi Ochiai, Department of Surgery, Tobu Chiiki Hospital, Tokyo Metropolitan Health and Medical Treatment Corporation, 5-14-1, Kameari, Katsushika, Tokyo 125-8512, Japan

E-mail: takumi-o@ma.kitanet.ne.jp

Key words: individualized chemotherapy, colorectal cancer, 5-fluorouracil, collagen gel droplet-embedded culture-drug sensitivity test, individual $\mathrm{AUC}_{\mathrm{IR} 50}$ the regression curve when the number of patients increased. A standard curve was obtained describing responders to 5-FU among all CRC patients. From this standard curve, we ascertained that non-responders accounted for approximately $5 \%$ of all patients. Moreover, we were able to classify responders into good or intermediate responders to 5-FU. The standard curve describing response to 5-FU in CRC patients offers a useful tool in the establishment of individualized chemotherapy.

\section{Introduction}

Over the past decade, individualized therapeutic strategies have been developed in the treatment of colorectal cancer (CRC). These strategies utilize a number of novel, molecularly targeted agents, yielding an improvement in survival (1-12). Despite an increase in our knowledge of molecular pathways, 40-50\% of metastatic CRC patients continue to experience no beneficial effect from such new strategies. This effect may be due to the numerous steps involved in the pharmacokinetics of anticancer drugs and various in vivo factors affecting sensitivity to anticancer drugs (13). Testing the chemosensitivity of cancer cells to a particular drug is therefore crucial in establishing which drug is appropriate.

As a key drug in the treatment of CRC, it is crucial to identify responders/non-responders to 5-fluorouracil (5-FU). Previously, we investigated the sensitivity of cancer cells to 5-FU in CRC patients using the collagen gel droplet-embedded culture-drug sensitivity test (CD-DST). Multiple drug concentrations and contact durations, and the area under the concentration curve (AUC) and growth inhibition rate (IR) were combined to produce an AUC-IR curve, which was approximated to the logarithmic curve. Moreover, the individualized $\mathrm{AUC}_{\mathrm{IR} 50}$ and $\mathrm{AUC}$ value, which gives $50 \%$ growth inhibition, were calculated using the AUC-IR curve $(14,15)$. This study aimed to identify responders/non-responders to 5-FU based on 
the individual $\mathrm{AUC}_{\mathrm{IR} 50}$ obtained with the CD-DST in order to establish individualized chemotherapy for CRC patients.

\section{Materials and methods}

Patients. Surgically resected specimens of primary tumors were obtained from 101 colorectal cancer patients between January 2002 and April 2010. Resection was performed without pre-operative chemotherapy. Informed consent for measuring drug sensitivity was obtained from the patients.

Methods. CD-DST was applied to tumor tissue excised from the primary surgical specimens. The specimen was washed five times with $50 \mathrm{ml}$ saline solution, followed by five times with $50 \mathrm{ml}$ antibiotic fluid containing $1.0 \mathrm{mg} / \mathrm{ml}$ piperacillin and $0.5 \mathrm{mg} / \mathrm{ml}$ kanamycin. The transport bottle contained $1.0 \mathrm{mg} / \mathrm{ml}$ piperacillin, $0.5 \mathrm{mg} / \mathrm{ml} \mathrm{kanamycin} \mathrm{and} 2.5 \mu \mathrm{g} / \mathrm{ml}$ amphotericin B. Tumor sensitivity to $5-\mathrm{FU}$ was evaluated using CD-DST, performed as described by Kobayashi et al (16). Tissue ( $1 \mathrm{~g})$ was treated with dispersion enzyme cocktail (EZ; Kurabo Industries Ltd., Japan) for 2 h. Dispersed cell suspensions were inoculated into pre-culture media in collagen-coated flasks overnight. Viable tumor cells were then recovered by $0.05 \%$ collagenase treatment. Recovered cells were embedded in 30- $\mu \mathrm{l}$ collagen gel droplets. Embedded cells were cultivated in culture media containing $5-\mathrm{FU}$ at $0.2 \mu \mathrm{g} / \mathrm{ml}$ for $3 \mathrm{~h}, 1 \mu \mathrm{g} / \mathrm{ml}$ for $3 \mathrm{~h}, 10 \mu \mathrm{g} / \mathrm{ml}$ for $3 \mathrm{~h}, 0.2 \mu \mathrm{g} / \mathrm{ml}$ for $24 \mathrm{~h}, 1$ $\mu \mathrm{g} / \mathrm{ml}$ for $24 \mathrm{~h}, 10 \mu \mathrm{g} / \mathrm{ml}$ for $24 \mathrm{~h}, 0.2 \mu \mathrm{g} / \mathrm{ml}$ for $120 \mathrm{~h}, 1 \mu \mathrm{g} / \mathrm{ml}$ for $120 \mathrm{~h}$ or $10 \mu \mathrm{g} / \mathrm{ml}$ for $120 \mathrm{~h}$. The 5 -FU-containing media were removed and the cells were cultured for 7 days in serumfree culture media (PCM-2; Kurabo Industries Ltd.) to prevent growth of fibroblasts. Viable cells were stained with neutral red solution and counted by the imaging colorimetric quantification method. The surviving cell number ratio between the drug-treated and control groups was calculated. Where a growth rate in excess of 0.8 was observed or $\geq 4$ culture conditions were recorded, the case was considered successful.

Following conversion of the drug concentrations and contact time to AUC, the AUC-IR curve was plotted against the growth inhibition rate and the individual $\mathrm{AUC}_{\mathrm{IR} 50}$, i.e., the $\mathrm{AUC}$ value that indicates $50 \%$ growth inhibition, was calculated from the AUC-IR regression curve. The approximate expression of the cumulative distribution of the individual $\mathrm{AUC}_{\mathrm{IR} 50}$ was obtained using the non-linear least squares method. The transition of the approximate expression with an increase in the number of patients was evaluated.

Statistical analysis. Correlations between the cumulative distribution of the individual $\mathrm{AUC}_{\mathrm{IR} 50}$ and the approximate expression of the cumulative distribution of the individual $\mathrm{AUC}_{\mathrm{IR} 50}$ were analyzed by linear regression analysis. The statistical tests were carried out using the SPSS package (version II for Windows). $\mathrm{P}<0.05$ was considered to be statistically significant.

\section{Results}

Table I shows the patient characteristics of the 101 patients enroled in this study. The AUC-IR curve of a representative patient is shown in Fig. 1.
Table I. Patient characteristics.

No. of patients

Mean age, years (range)

Gender (male/female)

$45 / 56$

Histological type

Well-differentiated carcinoma $\quad 15$

Moderately differentiated carcinoma $\quad 73$

Poorly differentiated carcinoma $\quad 4$

Mucinous carcinoma

9

Dukes' stage $(\mathrm{A} / \mathrm{B} / \mathrm{C} / \mathrm{D})$

$8 / 42 / 33 / 18$

Colon/rectum

$80 / 21$

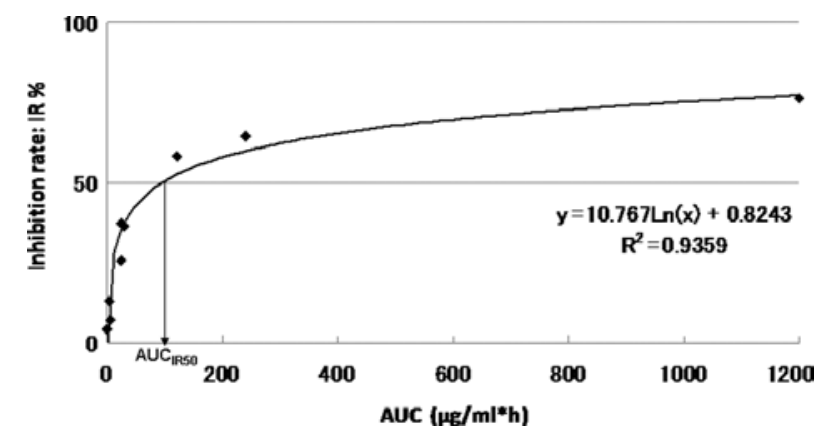

Figure 1. Growth inhibition according to the representative 5-fluorouracil area under curve (AUC); $\mathrm{AUC}_{\mathrm{IR} 50}$, AUC value imparting 50\% growth inhibition.

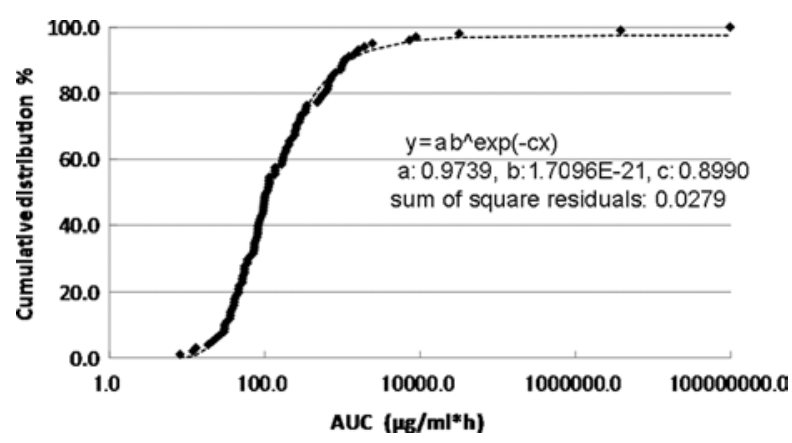

Figure 2. Cumulative distribution of individual area under curve $\mathrm{IR}_{\mathrm{IR}}$.

The approximate expression and correlation coefficients were $\mathrm{y}=10.767 \mathrm{Ln}(\mathrm{x})+0.8243,\left(\mathrm{R}^{2}=0.9359\right)$ for $\mathrm{AUC}$ and $96.3 \mu \mathrm{g} \mathrm{x} \mathrm{h} / \mathrm{ml}\left[\mathrm{y}=10.767 \operatorname{Ln}(\mathrm{x})+0.8243, \mathrm{R}^{2}=0.9359\right]$ for the individual $\mathrm{AUC}_{\mathrm{IR} 50}$ value calculated from the regression curve in this patient. The curve between the AUC and the growth IR approximated a logarithmic curve $\left(\mathrm{R}^{2}=0.655-0.999\right)$. The individual $\mathrm{AUC}_{\mathrm{IR} 50}$ value was calculated from the AUC-IR curve $\left(\mathrm{AUC}_{\mathrm{IR} 50}=8.1 \mu \mathrm{g}-98.8 \mathrm{~g} \mathrm{x} \mathrm{h} / \mathrm{ml}\right)$ for all 101 patients.

The cumulative distribution of the individual $\mathrm{AUC}_{\mathrm{IR} 50}$ in the 101 patients is shown in Fig. 2. The cumulative distribution of the individual $\mathrm{AUC}_{\mathrm{IR} 50}$ was regressed over the sigmoid curve (logarithmic scale). The approximate expression was almost exactly $\mathrm{y}=\mathrm{ab} \mathrm{b}^{\wedge} \exp (-\mathrm{cx}) \quad(\mathrm{a}=0.9739, \quad \mathrm{~b}=1.7096 \mathrm{E}-21$, $\mathrm{c}=0.8990$ and the sum of square residuals, 0.0279). 


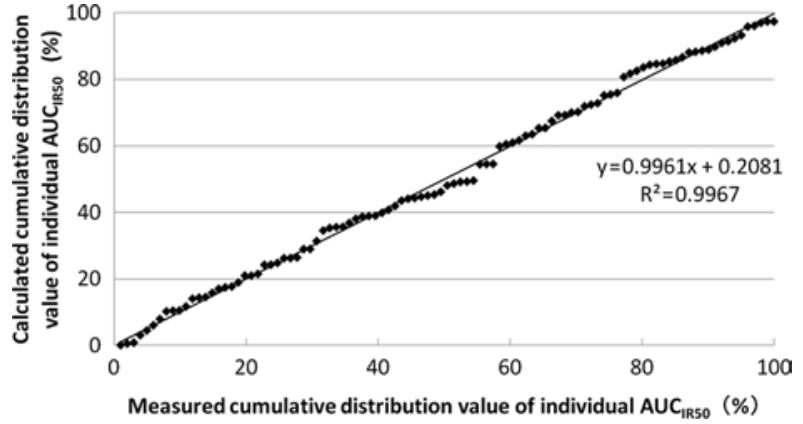

Figure 3. Correlation between measured and calculated cumulative distribution values of individual area under curve $\mathrm{IR}_{\mathrm{R} 50}$.

Table II. Linear regression with an increase in the number of patients.

\begin{tabular}{lccc}
\hline $\begin{array}{l}\text { No. of } \\
\text { patients }\end{array}$ & $\begin{array}{c}\text { Linear } \\
\text { regression }\end{array}$ & $\mathrm{R}^{2}$ & P-value \\
\hline 30 & $\mathrm{y}=0.9667 \mathrm{x}+0.0179$ & 0.9712 & 0.993 \\
40 & $\mathrm{y}=0.9717 \mathrm{x}+0.0162$ & 0.9824 & 0.979 \\
50 & $\mathrm{y}=0.9836 \mathrm{x}+0.0093$ & 0.9894 & 0.987 \\
60 & $\mathrm{y}=0.9918 \mathrm{x}+0.0042$ & 0.9918 & 1.000 \\
70 & $\mathrm{y}=0.9916 \mathrm{x}+0.0048$ & 0.9945 & 0.992 \\
80 & $\mathrm{y}=1.0005 \mathrm{x}-0.0011$ & 0.9954 & 0.985 \\
90 & $\mathrm{y}=0.9974 \mathrm{x}+0.0012$ & 0.9965 & 0.997 \\
101 & $\mathrm{y}=0.9961 \mathrm{x}+0.0021$ & 0.9967 & 0.998 \\
\hline
\end{tabular}

The correlation between the measured and the calculated cumulative distribution values of the individual $\mathrm{AUC}_{\mathrm{IR} 50}$ from the approximate expression is shown in Fig. 3. The approximate expression and correlation coefficients were $\mathrm{y}=0.9961 \mathrm{x}+0.2081$ $\left(\mathrm{R}^{2}=0.9967\right)$ using linear regression analysis. Linear regression with an increase in the number of patients is shown in Table II.

The transition of the coefficients ( $a, b$ and $c$ ) and the sum of the square residuals in the approximate expression $\left[y=a b^{\wedge} \exp (-\right.$ cx)] with an increase in the number of patients are shown in Figs. 4, 5, 6 and 7, respectively. In the 80 cases examined, no notable change was observed in the regression curve with an increase in the number of patients.

\section{Discussion}

Advances in the cytotoxic treatment of CRC have been noted over the past decade. Moreover, the introduction of novel molecularly targeted agents has yielded improvements in progression-free and overall survival in metastatic CRC patients (1-12). Despite these improvements in the treatment of $\mathrm{CRC}$ and increased knowledge regarding the molecular pathways involved, approximately half of all CRC patients have yet to receive any benefit from such progress, and selecting patients likely to prove responsive to a particular drug remains a challenge. Although a number of novel molecular predictive and prognostic markers have been identified (13), more remain to be determined, as numerous steps are involved in the

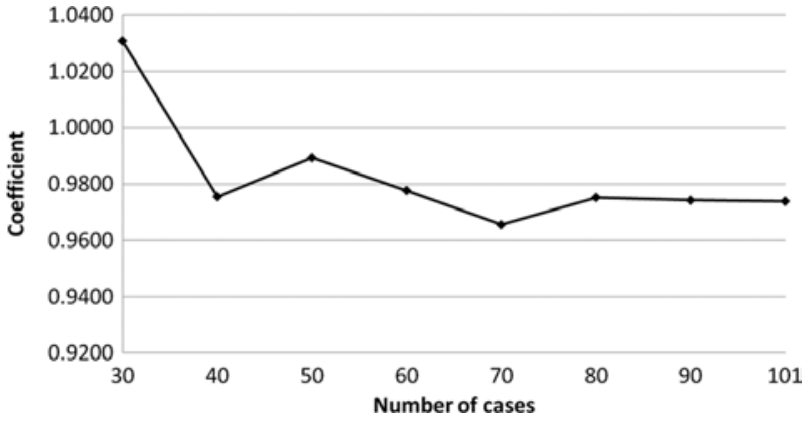

Figure 4. Transition of the coefficient (a) in the approximate expression $\left[y=a b^{\wedge} \exp (-c x)\right]$ with an increase in the number of patients.

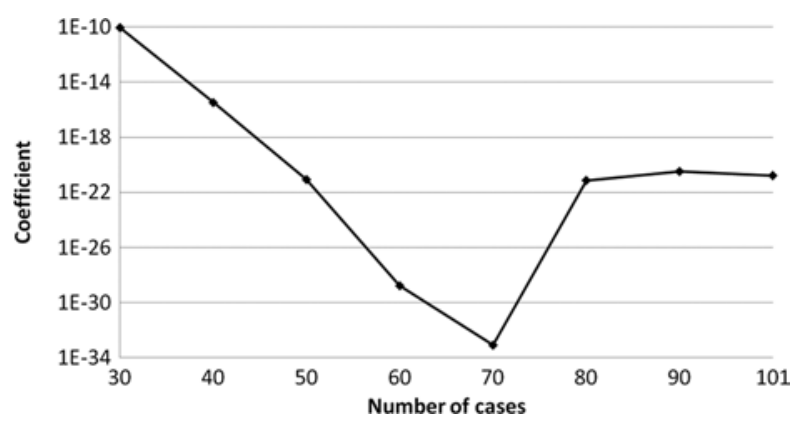

Figure 5. Transition of the coefficient (b) in the approximate expression $\left[y=a b^{\wedge} \exp (-c x)\right]$ with an increase in the number of patients.

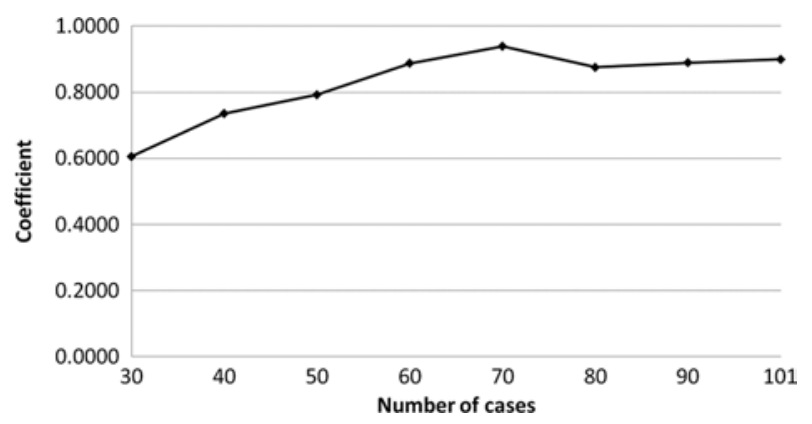

Figure 6. Transition of the coefficient (c) in the approximate expression $\left[y=a b^{\wedge} \exp (-c x)\right]$ with an increase in the number of patients.

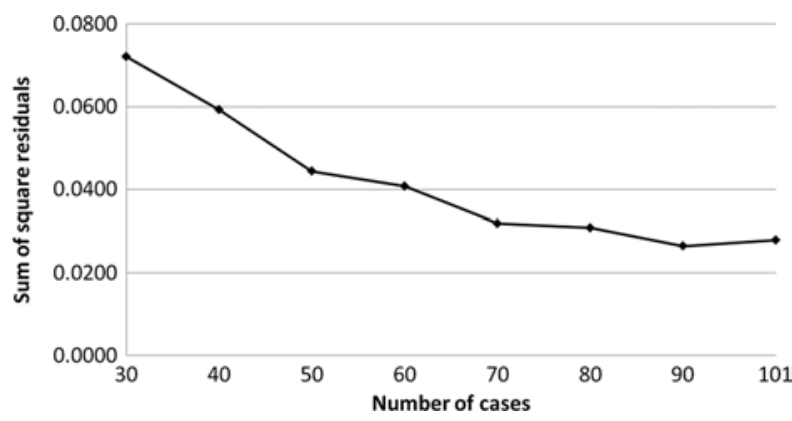

Figure 7. Transition of $x$ sum of square residuals in the approximate expression $\left[y=a b^{\wedge} \exp (-c x)\right]$ with an increase in the number of patients.

pharmacokinetics of anticancer drugs, especially with regard to absorption, metabolism and excretion. Numerous in vivo 
factors affect sensitivity to an anticancer drug, including activation enzymes, degradation enzymes, membrane transport proteins, DNA repair enzymes and the target protein in the cancer cell. These factors are regulated by individual genes. In other words, the behavior of the cancer is affected by the body as a whole. Thus, tumor and cell function, enzymatic activity, amount of enzymes, mRNA expression and, gene polymorphisms and mutations are involved in affecting the occurrence of cancer. Therefore, testing for chemosensitivity to a particular drug is crucial in distinguishing potential responders from non-responders.

The majority of chemotherapy regimens for CRC incorporate 5-FU, both in an adjuvant and palliative setting. 5-FU therefore is the key drug used in the treatment of CRC. The single-agent response rate to 5-FU varies between 20 and $25 \%$ in patients with advanced-stage CRC (17). No other anticancer agent surpasses 5-FU in terms of single-agent response rate. Therefore, assessing its antitumor effect in each individual CRC patient is crucial. Previously, we investigated cancer cell sensitivity to 5-FU in CRC patients using CD-DST under multiple drug concentrations and contact durations. Moreover, AUC and IR were combined to produce an AUC-IR curve, which was approximated to the logarithmic curve (14). We also reported that the growth inhibition rate calculated from the AUC-IR curve and the actual growth inhibition rate at an AUC of $48 \mu \mathrm{g} \mathrm{x} \mathrm{h/ml} \mathrm{were} \mathrm{identical.} \mathrm{Based} \mathrm{on} \mathrm{these} \mathrm{results,}$ we proposed that the in vitro antitumor effect of 5-FU was dependent on the AUC in colorectal cancer, and that the AUC-IR curve was reliable (15). Moreover, we calculated the individualized $\mathrm{AUC}_{\mathrm{IR} 50}$, the $\mathrm{AUC}$ value which gives $50 \%$ growth inhibition, using the AUC-IR curve (18).

In this study, we evaluated the distribution of the individual $\mathrm{AUC}_{\mathrm{IR} 50}$ in order to establish individualized chemotherapy in CRC patients, focusing, in particular, on the transition of the approximate expression with an increase in the number of patients. The cumulative distribution of the individual $\mathrm{AUC}_{\mathrm{IR} 50}$ in 101 patients was regressed over the sigmoid curve (logarithmic scale). The approximate expression was almost exactly $y=a b^{\wedge} \exp (-c x) \quad(a=0.9739, b=1.7096 E-21, c=0.8990$ and the sum of square residuals, 0.0279). The approximate expression and correlation coefficients between the measured and calculated cumulative distribution values of the individual $\mathrm{AUC}_{\mathrm{IR} 50}$ were $y=0.9961 x+0.2081\left(R^{2}=0.9967\right)$ using linear regression analysis. No notable change was observed in the transition of the coefficients (a,b and c) or the sum of the square residuals in the approximate expression $\left[y=a b^{\wedge} \exp (-c x)\right]$ with an increase in the number of patients. In particular, in over 80 cases the approximate expression $\left[y=a b^{\wedge} \exp (-c x)\right]$ remained almost identical. These results indicate that the regression curve may serve as the standard curve for describing responders to 5-FU among CRC patients. From this standard curve, we were able to ascertain that non-responders accounted for approximately $5 \%$ of all patients and, thus, determine the responders.

In individualized 5-FU-based chemotherapy for CRC, it is crucial to identify the non-responders and classify responders as good or intermediate by applying the standard curve to each patient. In palliative chemotherapy, irinotecan molecularly targeted drugs or irinotecan with molecularly targeted drugs are recommended for non-responders to 5-FU, whereas oxaliplatin plus 5 -FU and leucovorin (FOLFOX) \pm molecu- larly targeted drugs or irinotecan plus 5-FU and leucovorin $($ FOLFIRI \pm molecularly targeted drugs are recommended for responders (1-12). In adjuvant chemotherapy, good responders are suitable candidates for shorter-term, standard 5-FU-based chemotherapy, i.e., oral 5-FU or FOLFOX. Nevertheless, intermediate responders are also suitable candidates for longer-term or more intensive 5-FU-based chemotherapy, i.e., FOLFOX with molecularly targeted drugs. However, administration of molecularly targeted drugs in adjuvant chemotherapy remains to be investigated (19-21). Selection of adjuvant chemotherapy is challenging in non-responders to 5-FU. Single-response rates to irinotecan and oxaliplatin are relatively low; thus, close follow-up is required, and remains to be investigated.

More recently, numerous adjuvant chemotherapies have been assessed $(22,23)$. These adjuvant chemotherapy regimens have incorporated 5-FU, indicating the importance of evaluating the response to this drug. In the present study, a standard curve was generated to identify responders to 5-FU among CRC patients. From this standard curve, we were able to ascertain that non-responders accounted for approximately $5 \%$ of all patients. Moreover, we were able to identify responders as good or intermediate candidates. We believe that this standard curve offers a useful tool in identifying responders to 5-FU among CRC patients, enabling the establishment of individualized chemotherapy in CRC patients.

In Japan, the medical cost of CRC treatment, particularly in unresectable or recurring cases, is not cost-effective. Consequently, many patients are unable to receive such treatment due to the financial outlay involved. Therefore, our approach to identifying patients likely to be responsive to such treatment offers a viable solution.

\section{References}

1. De Gramont A, Figer A, Seymour M, et al: Leucovorin and fluorouracil with or without oxaliplatin as first-line treatment in advanced colorectal cancer. J Clin Oncol 18: 2938-2947, 2000.

2. Goldberg RM, Sargent DJ, Morton RF, et al: A randomized controlled trial of fluorouracil plus leucovorin, irinotecan, and oxaliplatin combinations in patients with previously untreated metastatic colorectal cancer. J Clin Oncol 22: 23-30, 2004.

3. Rothenberg ML, Oza AM, Bigelow RH, et al: Superiority of oxaliplatin and fluorouracil-leucovorin compared with either therapy alone in patients with progressive colorectal cancer after irinotecan and fluorouracil-leucovorin: interim results of a phase III trial. J Clin Oncol 21: 2059-2069, 2003.

4. Douillard JY, Cunningham D, Roth AD, et al: Irinotecan combined with fluorouracil compared with fluorouracil alone as first-line treatment for metastatic colorectal cancer: a multicentre randomised trial. Lancet 355: 1041-1047, 2000.

5. Tournigand $\mathrm{C}$, André $\mathrm{T}$, Achille E, et al: FOLFIRI followed by FOLFOX6 or the reverse sequence in advanced colorectal cancer: a randomized GERCOR study. J Clin Oncol 22: 229-237, 2004.

6. Hochster HS, Hart LL, Ramanathan RK, et al: Safety and efficacy of oxaliplatin and fluoropyrimidine regimens with or without bevacizumab as first-line treatment of metastatic colorectal cancer: results of the TREE Study. J Clin Oncol 21: 3523-3529, 2008.

7. Saltz LB, Clarke S, Díaz-Rubio E, et al: Bevacizumab in combination with oxaliplatin-based chemotherapy as first-line therapy in metastatic colorectal cancer: a randomized phase III study. J Clin Oncol 26: 2013-2019 2008.

8. Giantonio BJ, Catalano PJ, Meropol NJ, et al: Bevacizumab in combination with oxaliplatin, fluorouracil, and leucovorin (FOLFOX4) for previously treated metastatic colorectal cancer: results from the Eastern Cooperative Oncology Group Study E3200. J Clin Oncol 25: 1539-4154, 2007. 
9. Bokemeyer C, Bondarenko I, Makhson A, et al: Fluorouracil, leucovorin, and oxaliplatin with and without cetuximab in the first-line treatment of metastatic colorectal cancer. J Clin Oncol 27: 663-671 2009.

10. Maughan TS, Adams R, Smith CG, et al: Identification of potentially responsive subsets when cetuximab is added to oxaliplatin-fluoropyrimidine chemotherapy (CT) in first-line advanced colorectal cancer (aCRC). J Clin Oncol 28 (Suppl 5): abs. 3502, 2010.

11. Fuchs CS, Marshall J, Mitchell E, et al: Randomized, controlled trial of irinotecan plus infusional, bolus, or oral fluoropyrimidines in first-line treatment of metastatic colorectal cancer: results from the BICC-C Study. J Clin Oncol 25: 4779-4786, 2007.

12. Van Cutsem E, Köhne CH, Hitre E, et al: Cetuximab and chemotherapy as initial treatment for metastatic colorectal cancer. N Eng J Med 360: 1408-1417, 2009.

13. Winder $\mathrm{T}$ and Lenz HJ: Molecular predictive and prognostic marker in colon cancer. Cancer Treat Rev 36: 550-556, 2010.

14. Ochiai T, Nishimura K, Noguchi H, Kitajima M, Tsuruoka Y and Takahashi Y: Evaluation of 5-fluorouracil applicability by multi-point collagen gel droplet embedded drug sensitivity test. Oncol Rep 14: 201-205, 2005.

15. Ochiai $\mathrm{T}$, Nishimura $\mathrm{K}$, Noguchi $\mathrm{H}$, et al: Evaluation of 5 -fluorouracil applicability by the collagen gel droplet embedded drug sensitivity test with area under the curve analysis. Anticancer Drugs 18: 17-21, 2007.

16. Kobayashi H, Higashiyama M, Minamigawa $\mathrm{K}$, et al: Examination of in vitro chemosensitivity test using collagen ge droplet culture method with colorimetric endpoint quantification. Jpn J Cancer Res 92: 203-210, 2001.
17. Gill S, Loprinzi CL, Sargent DJ, et al: Pooled analysis of fluorouracil-based adjuvant therapy for stage II and III colon cancer: who benefits and by how much? J Clin Oncol 22: 1797-1806, 2004.

18. Ochiai T, Nishimura K, Watanabe T, et al: Evaluation of the individual $50 \%$ inhibitory area under the concentration curve of 5-fluorouracil based on the collagen gel droplet embedded culture drug sensitivity test in colorectal cancer. Mol Med Rep 2: 405-409, 2009

19. André T, Boni C, Mounedji-Boudiaf L, et al: Multicenter international study of oxaliplatin/5-fluorouracil/leucovorin in the adjuvant treatment of colon cancer (MOSAIC) investigators. N Eng J Med 350: 2343-2351, 2004

20. André T, Boni C, Navarro M, et al: Improved overall survival with oxaliplatin, fluorouracil, and leucovorin as adjuvant treatment in stage II or III colon cancer in the MOSAIC trial. J Clin Oncol 27: 3109-3116, 2009.

21. Wolmark N, Yothers G, O'Connell MJ, et al: A phase III trial comparing mFOLFOX6 to mFOLFOX6 plus bevacizumab in stage II or III carcinoma of the colon: results of NSABP Protocol C-08. J Clin Oncol 27 (Suppl 18): abs. LBA4, 2009.

22. Alberts SR, Sargent DJ, Smyrk TC, et al: Adjuvant mFOLFOX6 with or without cetuximab (Cmab) in KRAS wild-type (WT) patients (pts) with resected stage III colon cancer (CC): results from NCCTG Intergroup Phase III Trial NO 147. J Clin Oncol 28 (Suppl 18): abs. CRA3507, 2010.

23. Goldberg RM, Sargent DJ, Thibodeau SN, et al: Adjuvant mFOLFOX6 plus or minus cetuximab (Cmab) in patients (pts) with KRAS mutant $(\mathrm{m})$ resected stage III colon cancer (CC): NCCTG Intergroup Phase III Trial N0147. J Clin Oncol 28 (Suppl 15): abs. 3508, 2010. 